\title{
Motion Improvisation: 3D Human Motion Synthesis with a Transformer
}

\author{
Yimeng Liu \\ University of California, Santa Barbara \\ Santa Barbara, USA \\ yimengliu@cs.ucsb.edu
}

\author{
Misha Sra \\ University of California, Santa Barbara \\ Santa Barbara, USA \\ sra@cs.ucsb.edu
}

\begin{abstract}
The synthesis of complicated and realistic human motion is a challenging problem and is a significant task for game, film and animation industries. Many existing methods rely on complex and time-consuming keyframe-based methods that demand professional skills in animation software and motion capture hardware. On the other hand, casual users seek a playful experience to animate their favorite characters with a simple and easy-to-use tool. Recent work has explored building intuitive animation systems but suffers from inability to generate complex and expressive motions. To tackle this limitation, we present a keyframe-driven animation synthesis algorithm that is able to produce complex human motions with a few input keyframes, allowing the user to control the keyframes at will. Inspired by the success of attention-based techniques in natural language processing, our method completes body motions in a sequence-to-sequence manner and captures motion dependencies both spatially and temporally. We evaluate our method qualitatively and quantitatively on the LaFAN1 dataset, demonstrating improved accuracy compared with state of the art methods.
\end{abstract}

\section{CCS CONCEPTS}

- Computing methodologies $\rightarrow$ Neural networks; Motion processing.

\section{KEYWORDS}

Human motion synthesis, Machine learning, Keyframes, Animation

\section{ACM Reference Format:}

Yimeng Liu and Misha Sra. 2021. Motion Improvisation: 3D Human Motion Synthesis with a Transformer. In The Adjunct Publication of the 34th Annual ACM Symposium on User Interface Software and Technology (UIST '21 Adjunct), October 10-14, 2021, Virtual Event, USA. ACM, New York, NY, USA, 3 pages. https://doi.org/10.1145/3474349.3480219

\section{INTRODUCTION}

In the video game, film and animation industries, realistic performances for $\mathrm{CG}$ characters are in high demand. Conventional ways adopted by production studios and research labs to create realistic motion data include motion capture technologies and manual

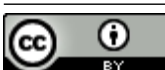

This work is licensed under a Creative Commons Attribution International 4.0 License.

UIST '21 Adjunct, October 10-14, 2021, Virtual Event, USA

(c) 2021 Copyright held by the owner/author(s).

ACM ISBN 978-1-4503-8655-5/21/10

https://doi.org/10.1145/3474349.3480219 keyframed animations. However, it is known that keyframe animation is time-consuming and requires professional skills [7]. Recent work has thus, explored easy-to-use interactive character animation builders. For example, Monster Mash [2] offers the technique and platform to generate casual animation based on hand-drawn curves. The disadvantage of these systems, however, is the inability to produce complicated and expressive animations that are difficult to describe with simple line-drawing representations.

Recent deep learning based techniques have demonstrated promising performance in automatically synthesizing multiple human motions with given keyframes, e.g., dancing [6], playing soccer [4], and action-conditioned motions [5]. However, these methods typically require task-specific goals or manual control of character locomotion. Other works have proposed techniques for generic motion generation $[3,7]$, but they usually fail to synthesize sophisticated human motions, e.g., fast forearm and leg movements. With the successful adoption of transformers in the field of computer vision, researchers have started to tackle the motion synthesis problem with self-attention provided by transformer encoding [1]. Although these works have shown superior performance in capturing longterm temporal attention of human motion, building an effective encoding of spatial structure is still an open research question.

In this work, we introduce an approach to address the aforementioned challenges and to synthesize human motion by achieving three main goals: 1) generate realistic and complex human motions, 2) precisely follow the given keyframes, and 3) demonstrate the potential for user control and interaction in the motion synthesis process. Our method is able to synthesize generic human motions and can be directly used to create animations for a variety of applications. By passing in varying density of keyframes at flexible space-time locations, our system can improvise a variety of motions under the keyframe constraints. To quantitatively evaluate our system, we compare with the SOTA method proposed by Duan, et al. [1], and demonstrate improved performance.

\section{METHOD}

We design the motion synthesis task as a sequence-to-sequence prediction problem conditioned on input keyframes. Our pipeline contains spatial attention and temporal attention to extract spatialtemporal correlations of bones in the human skeleton. They work holistically to synthesize human motion, both locally and globally. As a preliminary, the input of our system contains: local position and rotation + global position and rotation of each bone in a standard skeleton rig. Thus, we define the input as concatenated position variables $(x, y, z)$ and rotation variables $\left(r_{0}, r_{1}, r_{2}, r_{3}\right)$.

Spatial Attention. The novel component of our method is making use of a graph structure to dynamically update bone correlations. 
Specifically, in each frame, the spatial relationships of bones are learned with a graph convolution on a fully connected graph represented by a learnable adjacency matrix. We define the nodes as bones and the edges as their correlations. We set the initial edges of the graph as the physical connections of bones. During training, the graph is dynamically updated to extract body movement features.

Temporal Attention. Given the spatial features learned in each frame, the correlations of bones between frames are learned independently along the temporal dimension. The formulation is:

$$
\mathbf{z}_{i}^{v}=\sum_{j} \operatorname{softmax}_{j}\left(\frac{\mathbf{q}_{i}^{v} \cdot \mathbf{k}_{j}^{v}}{\sqrt{d_{k}}}\right) \mathbf{v}_{j}^{v}, \quad \forall v \in V
$$

where $\mathbf{q}_{i}^{v} \in \mathbb{R}^{d_{q}}$ is the query of bone $i^{v}, \mathbf{k}_{j}^{v} \in \mathbb{R}^{d_{k}}$ and $\mathbf{v}_{j}^{v} \in \mathbb{R}^{d_{v}}$ are the key and value pairs of a bone $j^{v}$ respectively. For each pair of bones along the temporal dimension, the query-key dot product is performed to get the weight expressing the strength of bone correlations in time. The weight is used to compute the weighted sum of value $\mathbf{v}_{j}^{v} \cdot z_{i}^{v}$ which is the resulting embedding of a bone $v$ at instant $i$. We then adopt a multi-head attention by repeating the embedding extraction of the same bone for $M$ times and combining the $M$ embeddings with a learnable transformation as the final output feature: $W_{o} \cdot \operatorname{concat}\left(\mathbf{z}_{i_{1}}^{v}, \ldots, \mathbf{z}_{i_{M}}^{v}\right)$.

Motion Prediction. Once the spatial-temporal features are extracted for each frame, they are fed into a feed-forward network (containing two linear layers and one GeLU layer) followed by a 1-D convolution layer to predict the human movements represented by positions and rotations locally and globally.

\subsection{User Control}

One interactive enabler of our system is flexible selection of keyframes decided by the user. Specifically, a creator may pick any frames with different poses to work as keyframes, and put them at intended spatial-temporal positions to expect complemented motions based on those limited keyframes produced by our system. Fig. 1 shows some possible generated movements given different keyframes. This feature opens up broad potential of our system to dynamically create various human motions easily.

\section{EVALUATION}

Dataset. We evaluate our method on the LaFAN1 dataset [3] and follow their train-test split protocol. This dataset contains 496,672 frames recorded from five subjects performing general motions, e.g., walking, dancing, jumping, etc.. The training set has motions from subjects 1 to 4 , while the testing set has motions from subject 5 .

Training and Testing. The LaFAN1 dataset consists of motions in long sequences, so we follow the strategy introduced in $[1,3]$ to partition the sequences according to motion windows. We set the window width as 50 frames and the window offset as 40 frames for both training and testing. We train our model for 200 epochs using a single GeForce RTX 2080 Ti 11GB graphics card.

\subsection{Motion Synthesis Results}

The human motion synthesis task is divided into two categories: in-betweening and in-filling, with qualitative results in Fig. 1.
3.1.1 In-betweening and In-filling. Motion in-betweening is the task of complementing missing frames between the given past and future keyframes. Results in Fig. 1b. Motion in-filling is an extension of in-betweening, where the selection of keyframes is more flexible. Based the motions posed at specific spatial-temporal positions, it fills in the rest of the frames. Results in Fig. 1c and $1 \mathrm{~d}$.

3.1.2 Analysis. From the results we can see that our method can properly complement human motions in missing frames following different number and position of keyframes. In the shown sequence, our method is able to complete some sophisticated movements like kicking and punching, and these fast and acute motions are challenging to generate using existing techniques.

Table 1: Evaluation results for L2P and L2Q (lower is better).

\begin{tabular}{|c|c|c|}
\hline Metric $\downarrow$ & L2P & L2Q \\
\hline Duan, et al. (local) & 1.37 & 0.71 \\
Ours (local) & 1.29 & 0.64 \\
\hline Duan, et al. (global) & 1.10 & 0.61 \\
Ours (global) & 1.11 & 0.53 \\
\hline
\end{tabular}

\subsection{Comparison with State of the Art}

3.2.1 Metrics. Following [1], we use L2P and L2Q as our evaluation metrics. L2P evaluates the mean Euclidean distances of the global positions between the ground-truth and the synthesized motions. L2Q measures the mean Euclidean distances of the global quaternions between the ground-truth and the synthesized motions.

3.2.2 Baseline Method \& Performance. We compare with SOTA algorithm by Duan, et al. [1]. For a fair comparison, we keep the same inference configuration as the protocol used in [1], and report the evaluation results in Table. 1 in both local and global coordinates. We see in Table. 1 that the accuracy of both position and rotation predictions is improved especially in the local coordinate system, whereas Duan, et al. [1] found that "the motion synthesis task can be better addressed in the global coordinate system since the errors of rotations in local coordinates can easily accumulate". This performance demonstrates the effectiveness of spatial attention encoded by the graph structure introduced in our model and offers a possible solution to address the local motion synthesis problem.

\section{CONCLUSION AND DISCUSSION}

In this work, we proposed a spatial-temporal encoding method using a graph structure to synthesize generic and realistic human motions precisely following given keyframes at flexible space-time locations. We evaluated our algorithm on the public LaFAN1 dataset, and demonstrated improved motion completion accuracy and plausible motion sequences under multiple keyframe constraints.

Our method offers a solution to generate complex human motions, but limited by the dataset we use, delicate motions, e.g., hand and finger movements, are unstudied. We plan to evaluate our technique on other mocap datasets to test generalization and enhance fine-granularity of synthesized motions. Additionally, we plan to expand our method to quadruped skeletons and fantasy creatures with unusual body types, that are under-explored in prior work. 


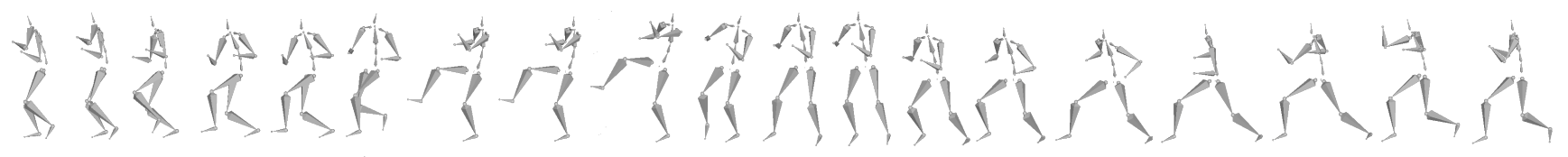

(a) Ground-truth motion sequence.

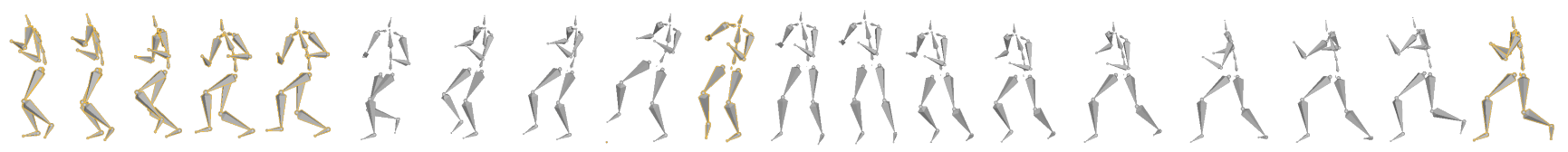

(b) In-betweening results.

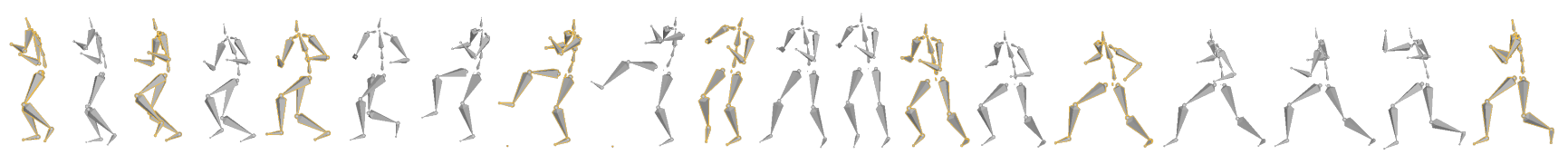

(c) In-filling results with dense keyframes.

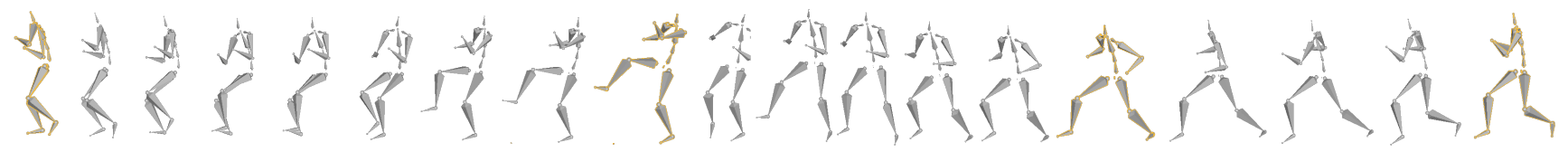

(d) In-filling results with sparse keyframes.

Figure 1: Motion Improvisation Results. (a) is the ground-truth motion sequence, where the movements contain kicking and punching, (b) shows the in-betweening results based on the first five frames, a random chosen keyframe and the last frame, (c) and (d) are in-filling results based on random chosen dense and sparse keyframes, respectively.

\section{REFERENCES}

[1] Yinglin Duan, Tianyang Shi, Zhengxia Zou, Yenan Lin, Zhehui Qian, Bohan Zhang, and Yi Yuan. 2021. Single-Shot Motion Completion with Transformer. arXiv preprint arXiv:2103.00776 (2021).

[2] Marek Dvorožňák, Daniel Sỳkora, Cassidy Curtis, Brian Curless, Olga SorkineHornung, and David Salesin. 2020. Monster mash: a single-view approach to casual 3D modeling and animation. ACM Transactions on Graphics (TOG) 39, 6 (2020), 1-12.

[3] Félix G Harvey, Mike Yurick, Derek Nowrouzezahrai, and Christopher Pal. 2020. Robust motion in-betweening. ACM Transactions on Graphics (TOG) 39, 4 (2020), $60-1$.
[4] Seokpyo Hong, Daseong Han, Kyungmin Cho, Joseph S Shin, and Junyong Noh. 2019. Physics-based full-body soccer motion control for dribbling and shooting. ACM Transactions on Graphics (TOG) 38, 4 (2019), 1-12.

[5] Mathis Petrovich, Michael J Black, and Gül Varol. 2021. Action-Conditioned 3D Human Motion Synthesis with Transformer VAE. arXiv preprint arXiv:2104.05670 (2021).

[6] Benedikte Wallace, Charles P Martin, Jim Tørresen, and Kristian Nymoen. 2021. Learning Embodied Sound-Motion Mappings: Evaluating AI-Generated Dance Improvisation. In Creativity and Cognition. 1-9.

[7] Yi Zhou, Jingwan Lu, Connelly Barnes, Jimei Yang, Sitao Xiang, et al. 2020. Generative Tweening: Long-term Inbetweening of 3D Human Motions. arXiv preprint arXiv:2005.08891 (2020). 